hypotension, bradycardia and arrhythmia) of sedation during ESD were evaluated between two groups.

Results Propensity score matching analysis created 91 matched pairs. Adjusted comparisons between Group E and O showed similar treatment outcomes of ESD (en bloc resection rate: $98.9 \%$ vs. $100 \%, \mathrm{p}=1.000$; perforation rate: $4.4 \%$ vs. $1.1 \%$, $\mathrm{p}=0.368$; delayed haemorrhage rate: $8.8 \%$ vs. $2.2 \%$, $\mathrm{p}=0.100)$. In adverse events, desaturation occurred significantly more often in Group E than $\mathrm{O}(16.5 \%$ vs. $3.3 \%$, $\mathrm{p}=0.005)$. There were no significant differences in other adverse events between the two groups (hypotension rate: $13.2 \%$ vs. $7.7 \%, \mathrm{p}=0.333$, bradycardia rate: $5.5 \%$ vs. $1.1 \%$, $\mathrm{p}=0.211$, and arrhythmia rate: $0 \%$ vs. $0 \%, \mathrm{p}=1.000$ ).

Conclusions Whereas a decrease in the desaturation rate was the only advantages of sedation with propofol in the operation room, sedation in the operation room might be required to ensure safer application of ESD for gastric tumours.

\section{IDDF2018-ABS-0075 MANAGEMENT OF ANAEMIA IN INFLAMMATORY BOWEL DISEASE}

Hang Hoi Michael Wong*, Ying Ki Shirley Tze. United Christian Hospital, Hong Kong

\subsection{6/gutjnl-2018-IDDFabstracts.99}

Background Anaemia is the most common extraintestinal manifestation of IBD. It has a substantial impact on patients' morbidities and healthcare costs.

Methods We conducted a retrospective observational study on all our patients (total numbers: 224) suffering from IBD to determine whether anaemia was addressed during their clinic visits and whether treatment(s) were appropriately given. The haemoglobin, mean cell volume (MCV), ferritin, total iron binding capacity (TIBC), iron saturation (in\%) and CRP were reviewed.

Results Among the total 132 (59\%) Ulcerative colitis (UC) patients and 92 (41\%) Crohn's disease (CD) patients, 91 (40\%) patients suffered from anaemia at diagnosis. $93(42 \%)$ patients had ferritin, TIBC and iron saturation reviewed and documented. $71(32 \%)$ patients received iron supplements at some point since the diagnosis of IBD was made. All patients with iron deficiency anaemia were correctly identified and were offered iron supplements. However, of those suffered from iron deficiency anaemia, only 24 (34\%) patients had their ferritin, TIBC and iron saturation monitored every three months after adequate replacement to prevent recurrent iron deficiency. 25/91 (28\%) of IBD patients suffered from noniron deficiency anaemia (NIDA): 9 patients suffered from B12 deficiency and were all offered a parenteral replacement, 16 patients were given the diagnosis of anaemia of chronic disease (ACD), who demonstrated clinical or biochemical evidence of inflammation. During their most recent clinic visits, all patients had their haemoglobin reviewed and 47 (21\%) patients still suffered from anaemia. 10/47 (22\%) were noncompliant with their iron supplements; 16/47 (34\%) were due to active inflammation; the rest did not have a clear diagnosis.

Conclusions The aetiology of anaemia in IBD patients were mainly categorised into iron deficiency anaemia and NIDA. Adequate iron replacement and compliance are important in the treatment of iron deficiency. Recurrence of iron deficiency can be prevented by monitoring blood counts, ferritin, TIBC and iron saturation every three to six months. NIDA requires further work-up, and optimisation of IBD treatment should be first considered especially in ACD. In patients with persistent anaemia despite iron replacement and optimised IBD treatment, one should consider the use of erythropoiesis-stimulating agents.

\section{IDDF2018-ABS-0084 RECURRENT ACUTE PANCREATITIS IN A CHILD - AETIOLOGY BEING A RARE PANCREATIC DUCT ANOMALY}

Varun Palanati*. Narayana Medical College, Nellore, India

\subsection{6/gutjnl-2018-IDDFabstracts.100}

Background Acute pancreatitis is an emerging problem in paediatrics. Although the cause is unclear, it may be explained by a heightened awareness of AP in children. Although magnetic resonance cholangiopancreatography (MRCP) is seldom required for the first attack of $\mathrm{AP}$, it constitutes a valuable tool in the evaluation of pancreaticobiliary abnormalities. Congenital anomalies and normal variants of the pancreatic duct are often detected as incidental findings in asymptomatic patients. Occasionally it produces symptoms in childhood and here is a patient presented to us with recurrent episodes of abdominal pain and MRCP helped us in diagnosing the condition and directed us for therapy.

Methods 13-year-old male presented to us with complaints of upper abdominal pain since 5 days, vomitings since 4 days. His abdominal pain is radiating to back. He had similar episodes for three times in the past 2 years but were treated conservatively at a local hospital and not been investigated. On examination, vitals were stable and abdominal examination was normal. In view of the history of recurrent acute pancreatitis, MRCP was done. It showed bifid duct of wirsung with duct of santorini opening into its anterior division with bulky body and tail of the pancreas. The patient was conservatively treated, and he recovered well

Results It was a case report showing pancreatic duct anomaly causing recurrent acute pancreatitis.

Conclusions Congenital variants of the biliopancreatic ductal system provide interesting challenges when discovered during the diagnostic workup of idiopathic acute recurrent pancreatitis. However, most of these variants are clinically irrelevant. Methods for the selection of patients most likely to benefit from invasive therapy need to be refined.

\section{IDDF2018-ABS-0086 NON-ALCOHOLIC FATTY PANCREAS DISEASE AS AN INDEPENDENT PREDICTOR OF ACUTE PANCREATITIS: A RETROSPECTIVE STUDY OF 264 PATIENTS}

${ }^{1}$ Jiarong Xie*, ' Lei Xu. 'Ningbo University, College of Medicine, Ningbo, China; Department of Gastroenterology, Ningbo First Hospital, Ningbo, China; ${ }^{2}$ Department of Gastroenterology, Ningbo First Hospital, Ningbo, China

\subsection{6/gutjnl-2018-IDDFabstracts. 101}

Background This study is aimed to investigate the relationship between non-alcoholic fatty pancreas disease (NAFPD) and the severity of acute pancreatitis (AP) and to assess the significance of pancreas attenuation in the prognosis and mortality of AP patients. 